\title{
Patient with juvenile rheumatoid arthritis submitted to cornea transplant - solution for the difficult airway.
}

K. Czajkowska*, M. Bela, L. Germanova; Coimbra Hospital and University Centre

Euroanaesthesia

GENEVA 03-05 JUNE

THE EUROPEANANAESTHESIOLOG CONGRESS

\section{Background}

Juvenile rheumatoid arthritis (RA) is a chronic autoimmune multisystemic inflammatory disease which affects many organs but predominantly attacks joints. In many patients it can cause movement limitations of

the neck and the temporomandibular joint leading to a difficult airway. The use of supraglottic airway devices (SADs) presents many advantages in patients with RA scheduled for an elective ophthalmic surgery

\section{Case report:}

5 I years old female, $40 \mathrm{~kg}$ and I,3m (BMI 23,4m²/kg), with advanced juvenile RA, completely dependent on activities of daily living, who due to cornea transplant failure was scheduled for a reintervention. Preoperative evaluation revealed multiple osteoarticular deformities of upper and lower limbs, severe kyphoscoliosis, reduced cervical mobility, thyromental distance $<6 \mathrm{~cm}$, mouth opening around $3 \mathrm{~cm}$ and class IV Mallampati. Previous procedure was performed under general anaesthesia and fiberscope was necessary for the endotracheal intubation. We proceeded with the induction of anaesthesia with $8 \%$ Sevoflurane and when the loss of consciousness was achieved, we secured the airway with a SAD. l-gel device $n^{\circ} 3$ was inserted with no difficulties and a good seal was obtained. During the induction phase, fiberscope was immediately accessible and could be used if necessary. Maintenance of anaesthesia was continued with $2 \%$ Sevoflurane, patient was mechanically ventilated and we reported no complications during the surgery nor during the extubation.

\section{Discussion:}

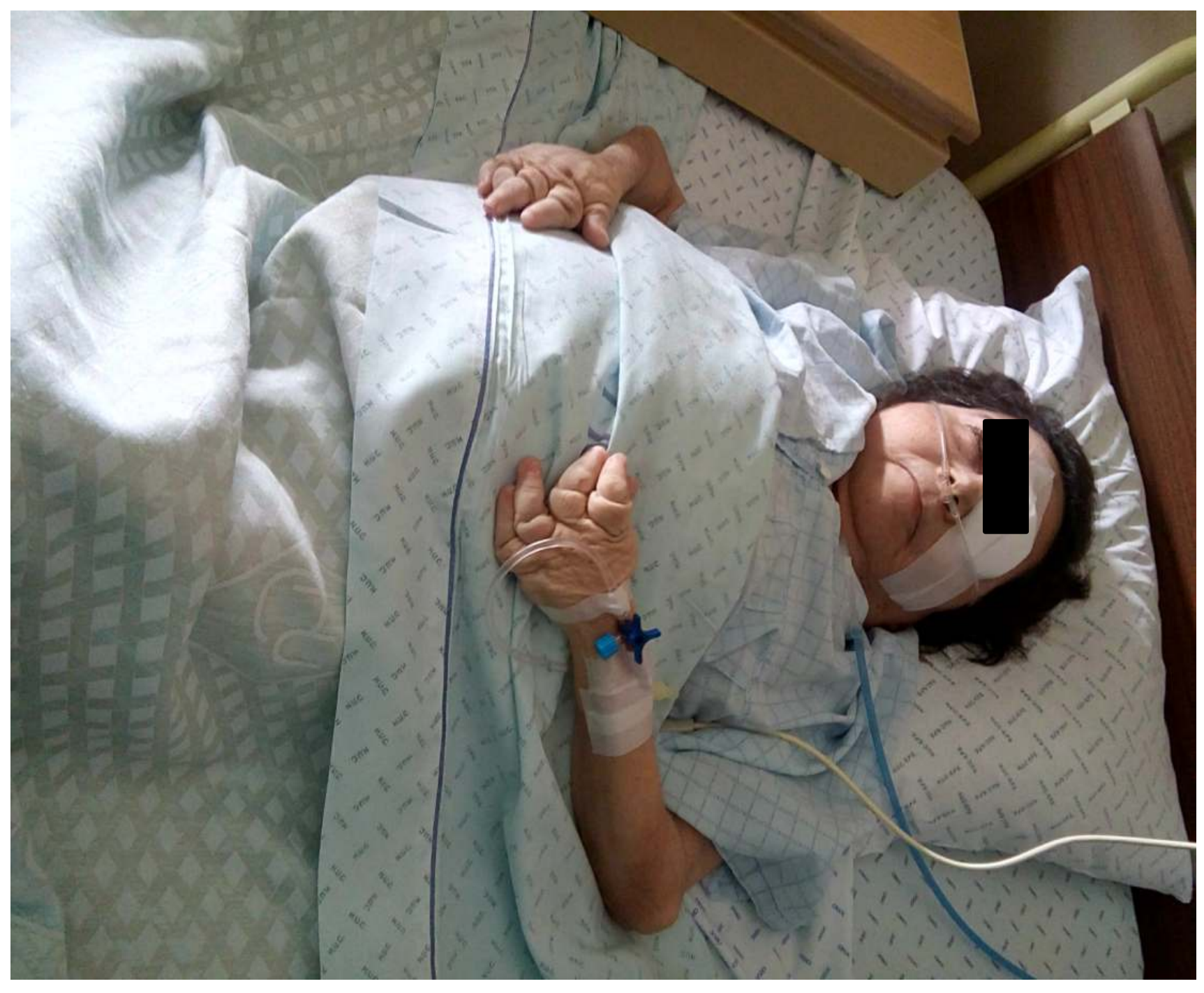

Due to severe deformities, anaesthesia management of that patient was challenging. With a predicted difficult airway, in the context of ophthalmic surgery, SAD may be indicated. MLA may help to avoid the increase of the intra-ocular pressure related to endotracheal intubation or coughing during the extubation, it also provides a smoother recovery ${ }^{2}$. To conclude, SAD may be used as a primary approach in patients with predicted difficult airway due to juvenile RA, scheduled for an ophthalmic surgery but access to fiberscope should be secured.

\section{References:}

